\title{
Convergent losses of decay mechanisms and rapid turnover of symbiosis genes in mycorrhizal mutualists
}

\author{
Annegret Kohler $1,2,25$, Alan Kuo ${ }^{3,25}$, Laszlo G Nagy, 5,25, Emmanuelle Morin ${ }^{1,2,25}$, Kerrie W Barry ${ }^{3}$, \\ Francois Buscot ${ }^{6,7}$, Björn Canbäck ${ }^{8}$, Cindy $\mathrm{Choi}^{3}$, Nicolas Cichocki ${ }^{1,2}$, Alicia Clum ${ }^{3}$, Jan Colpaert ${ }^{9}$, \\ Alex Copeland ${ }^{3}$, Mauricio D Costa ${ }^{10}$, Jeanne Doré ${ }^{11}$, Dimitrios Floudas ${ }^{4}$, Gilles Gay ${ }^{11}$, Mariangela Girlanda ${ }^{12}$, \\ Bernard Henrissat $^{13-15}$, Sylvie Herrmann ${ }^{6,7}$, Jaqueline Hess ${ }^{16}$, Nils Högberg ${ }^{17}$, Tomas Johansson ${ }^{8}$, \\ Hassine-Radhouane Khouja ${ }^{12}$, Kurt LaButti ${ }^{3}$, Urs Lahrmann ${ }^{18}$, Anthony Levasseur ${ }^{13}$, Erika A Lindquist ${ }^{3}$, \\ Anna Lipzen 3 , Roland Marmeisse ${ }^{11}$, Elena Martino ${ }^{1,12}$, Claude Murat ${ }^{1,2}$, Chew Y Ngan ${ }^{3}$, Uwe Nehls ${ }^{19}$, \\ Jonathan M Plett ${ }^{1,2}$, Anne Pringle ${ }^{20}$, Robin A Ohm ${ }^{3}$, Silvia Perotto ${ }^{12}$, Martina Peter ${ }^{21}$, Robert Riley ${ }^{3}$, \\ Francois Rineau, ${ }^{9,24}$, Joske Ruytinx ${ }^{9}$, Asaf Salamov ${ }^{3}$, Firoz Shah ${ }^{8}$, Hui Sun ${ }^{3}$, Mika Tarkka ${ }^{6,7}$, Andrew Tritt ${ }^{3}$, \\ Claire Veneault-Fourrey ${ }^{1,2}$, Alga Zuccaro ${ }^{18,22}$, Mycorrhizal Genomics Initiative Consortium ${ }^{23}$, Anders Tunlid ${ }^{8}$, \\ Igor V Grigoriev ${ }^{3,26}$, David S Hibbett ${ }^{4,26}$ \& Francis Martin ${ }^{1,2,26}$
}

To elucidate the genetic bases of mycorrhizal lifestyle evolution, we sequenced new fungal genomes, including 13 ectomycorrhizal (ECM), orchid (ORM) and ericoid (ERM) species, and five saprotrophs, which we analyzed along with other fungal genomes. Ectomycorrhizal fungi have a reduced complement of genes encoding plant cell walldegrading enzymes (PCWDEs), as compared to their ancestral wood decayers. Nevertheless, they have retained a unique array of PCWDEs, thus suggesting that they possess diverse abilities to decompose lignocellulose. Similar functional categories of nonorthologous genes are induced in symbiosis. Of induced genes, $7-38 \%$ are orphan genes, including genes that encode secreted effector-like proteins. Convergent evolution of the mycorrhizal habit in fungi occurred via the repeated evolution of a 'symbiosis toolkit', with reduced numbers of PCWDEs and lineage-specific suites of mycorrhiza-induced genes.
Fungi are often described as either saprotrophs, which degrade complex organic substrates, or biotrophs, which obtain carbon compounds from living hosts. Among the latter, ECM fungi provide crucial ecological services in interacting with forest trees. They are portrayed as mutualists trading host photoassimilates for nutrients and having limited capacity to decompose soil lignocellulose ${ }^{1-3}$, as a result of their reduced repertoire of PCWDEs ${ }^{4-6}$. However, recent studies are challenging this view ${ }^{7-10}$. An improved understanding of the ability of ECM fungi to decompose lignocellulose is needed to resolve mechanisms of nutrient cycling in forests. The ECM lifestyle in Laccaria bicolor is associated with the expression of new mycorrhizainduced small secreted proteins (MiSSPs) that are required for establishment of symbiosis ${ }^{11,12}$. Mycorrhizal symbioses have arisen repeatedly during fungal evolution and include not only ECM associations but also those with ERM and ORM mycorrhizae ${ }^{13}$. It is not known whether these symbioses share the genomic features found in L. bicolor ${ }^{4}$ and Tuber melanosporum ${ }^{5}$. Here we assess whether there

${ }^{1}$ Institut National de la Recherche Agronomique (INRA), Laboratory of Excellence Advanced Research on the Biology of Tree and Forest Ecosystems (ARBRE), UMR 1136, Champenoux, France. 2University of Lorraine, Laboratory of Excellence ARBRE, UMR 1136, Champenoux, France. ${ }^{3}$ US Department of Energy Joint Genome Institute (JGI), Walnut Creek, California, USA. ${ }^{4}$ Department of Biology, Clark University, Worcester, Massachusetts, USA. ${ }^{5}$ Synthetic and Systems Biology Unit, Institute of Biochemistry, Biological Research Center, Hungarian Academy of Sciences, Szeged, Hungary. ${ }^{6}$ Department of Soil Ecology, Helmholtz Centre for Environmental Research-Helmholtz Zentrum fuer Umweltforschung, Halle, Germany. ${ }^{7}$ German Centre for Integrative Biodiversity Research (iDiv) Halle-Jena-Leipzig, Leipzig, Germany. ${ }^{8}$ Department of Biology, Microbial Ecology Group, Lund University, Lund, Sweden. ${ }^{9}$ Centre for Environmental Sciences, Hasselt University, Diepenbeek, Belgium. ${ }^{10}$ Departamento de Microbiologia, Bolsista do Conselho Nacional de Desenvolvimento Científico e Tecnológico (CNPq), Universidade Federal de Viçosa, Viçosa, Brazil. ${ }^{11}$ UMR CNRS 5557, Unité Sous Contrat INRA 1364, Université de Lyon, Université Lyon 1, Villeurbanne, France. ${ }^{12}$ Dipartimento di Scienze della Vita e Biologia dei Sistemi, Università degli Studi di Torino, Torino, Italy. ${ }^{13}$ CNRS, UMR 7257, Aix-Marseille Université, Marseille, France. ${ }^{14}$ Architecture et Fonction des Macromolécules Biologiques, Aix-Marseille Université, Marseille, France. ${ }^{15}$ Department of Biological Sciences, King Abdulaziz University, Jeddah, Saudi Arabia. ${ }^{16}$ Department of Biosciences, University of Oslo, Oslo, Norway. ${ }^{17}$ Department of Forest Mycology and Pathology, Swedish University of Agricultural Sciences, Uppsala, Sweden. ${ }^{18}$ Department of Organismic Interactions, Max Planck Institute for Terrestrial Microbiology, Marburg, Germany. ${ }^{19}$ Department of Ecology, Biology/Chemistry, Botany, University of Bremen, Bremen, Germany. ${ }^{20}$ Harvard Forest, Harvard University, Petersham, Massachusetts, USA. ${ }^{21}$ Swiss Federal Institute for Forest, Snow and Landscape Research (WSL), Forest Dynamics, Birmensdorf, Switzerland. 22University of Cologne, Botanical Institute, Cluster of Excellence on Plant Sciences (CEPLAS), Cologne, Germany. ${ }^{23} \mathrm{~A}$ full list of members is provided in the Supplementary Note. ${ }^{24}$ Present address: Institut de Systématique, Évolution, Biodiversité (ISYEB), UMR 7205, CNRS, Muséum National d'Histoire Naturelle, Université Pierre et Marie Curie, Ecole Pratique des Hautes Etudes, Sorbonne Universités, Paris, France. ${ }^{25}$ These authors contributed equally to this work. ${ }^{26}$ These authors jointly supervised this work. Correspondence should be addressed to F.M. (fmartin@nancy.inra.fr) or D.S.H. (DHibbett@clarku.edu) 
Figure 1 Evolution of mycorrhizal symbiosis inferred from 49 fungal genomes. The tree is a chronogram estimated with r8s on the basis of a maximum-likelihood phylogeny inferred with RAXML. Nodes receiving less than maximal support in all analyses are indicated with asterisks. Curved arrows indicate alternate placements for Ustilaginomycotina and Auriculariales (Supplementary Fig. 2). Mean ages (ma) are indicated adjacent to selected nodes. Circles indicate observed (right of tree) and reconstructed (left) copy numbers for selected genes encoding enzymes involved in decay of lignin (POD and GLX; blue circles) or crystalline cellulose (GH6, GH7 and LPMO; beige circles). Absence of gene copies is indicated with ' $x$ '. Areas of circles are proportional to gene copy numbers. (Copy numbers are indicated for internal nodes; gene counts in terminal taxa are shown in Supplementary Table 9.) Selected clades are labeled at internal nodes; 'st.' indicates the stem node for a taxon. Shading of terminal taxon names indicates nutritional modes (as shown in the key). Solid red triangles, estimated origins of ECM or ERM and ORM mycorrhizal symbioses; unfilled red triangle, alternate reconstruction with a single origin of ECM in Boletales and at least one reversal to saprotrophy; colored triangles below the geological timescale, ages of major ECM hosts based on fossils (solid triangles) and light-gray shading, temporal period when Orch/eric/endomyc, orchid, ericoid or endomycorrhizae; litter/soil/other, litter, soil or other saprotroph; Cryog., Cryogenian; Ediac., Ediacaran; Cam., Cambrian; Ord., Ordovician; Sil., Silurian; Dev., Devonian; Carb., Carboniferous; Per., Permian; Tri., Triassic; Jur., Jurassic; Cret., Cretaceous; Cen., Cenozoic. Abbreviations for taxon names are defined in Supplementary Note. molecular-clock estimates (unfilled triangles); the origins of ECM are most plausible.

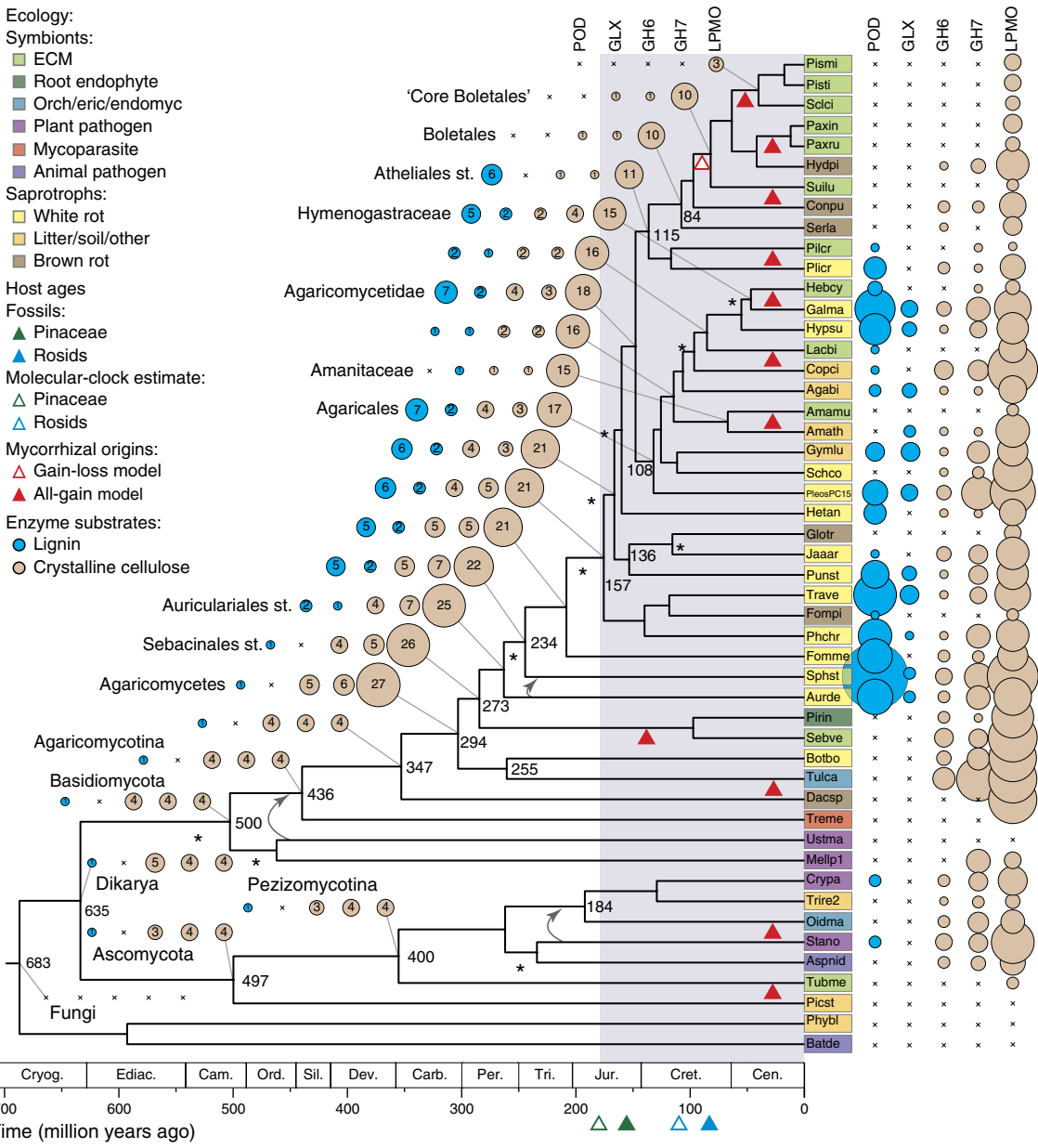

are general evolutionary and functional properties of mycorrhizal Basidiomycota and Ascomycota, using comparative analysis of 18 newly sequenced genomes (Supplementary Tables 1 and 2), including 13 from ECM, ERM and ORM fungal symbionts.

Genomes of mycorrhizal fungi range in size from approximately 38 to $125 \mathrm{Mb}$, and their predicted gene contents range from approximately 15,000 to 23,000 genes (Supplementary Tables 3 and 4). We combined the 18 new genomes with 31 others and performed phylogenomic analyses (Supplementary Note, Supplementary Tables 1 and 5-10, and Supplementary Figs. 1-23). An organismal phylogeny resolved six to eight independent lineages of ECM and two lineages of ORM symbionts in Agaricomycetes as well as two mycorrhizal lineages in Ascomycota (Fig. 1). Molecular-clock analyses have suggested that the Pezizomycotina and Agaricomycetes are approximately 400 and 300 million years old, respectively. In contrast, the Pinaceae (which may be the oldest ECM hosts), rosids and orchids are of Jurassic or Cretaceous origin ( 100 million years ago), according to fossil and molecular-clock evidence; this is consistent with the view that ECM and other mycorrhizal associations have evolved repeatedly across Dikarya ${ }^{14}$.

The first three sequenced mycorrhizal genomes, i.e., the ECM L. bicolor and T. melanosporum $m^{4,5}$ and the arbuscular mycorrhizal fungus Rhizophagus irregularis ${ }^{15}$, demonstrated substantial losses in PCWDE genes. To assess the diversity of lignocellulose-decay capabilities of other mycorrhizal lineages, we cataloged 28 gene families and subfamilies encoding oxidoreductases and carbohydrateactive enzymes (CAZymes) associated with plant cell wall degradation (Fig. 2, Supplementary Note, Supplementary Tables 7-10 and Supplementary Fig. 3). Among Agaricomycetes, saprotrophs (including white-rot decayers, which can degrade lignin as well as cellulose), Botryobasidium botryosum, Jaapia argillacea and soil and litter saprotrophs possess on average 133 gene copies across all PCWDE families, whereas brown-rot species (which lack the ability to degrade lignin) possess 81 gene copies, and ECM species maintain 62 gene copies, on average. Thus, the evolution of ECM parallels that of brownrot lineages, with both guilds having lost much of the plesiomorphic enzymatic apparatus of white rots (Supplementary Note).

To reconstruct the evolution of decay capabilities, we performed gene tree-species tree reconciliation for 16 gene families encoding PCWDEs (Supplementary Note and Supplementary Figs. 4-23). Here we focus on enzymes acting on lignin and crystalline cellulose, including class II peroxidases (PODs), glyoxal oxidase (GLX; an accessory enzyme that generates $\mathrm{H}_{2} \mathrm{O}_{2}$ as a substrate for PODs), GH6 and GH7 (cellobiohydrolases) and lytic polysaccharide monooxygenases (LPMOs) (Supplementary Figs. 4-23). Expansion of LPMO genes, from 4 copies in the ancestor of Basidiomycota to 27 copies in the ancestor of Agaricomycetes, preceded the initial duplication of ligninolytic POD genes and the origin of the GLX 


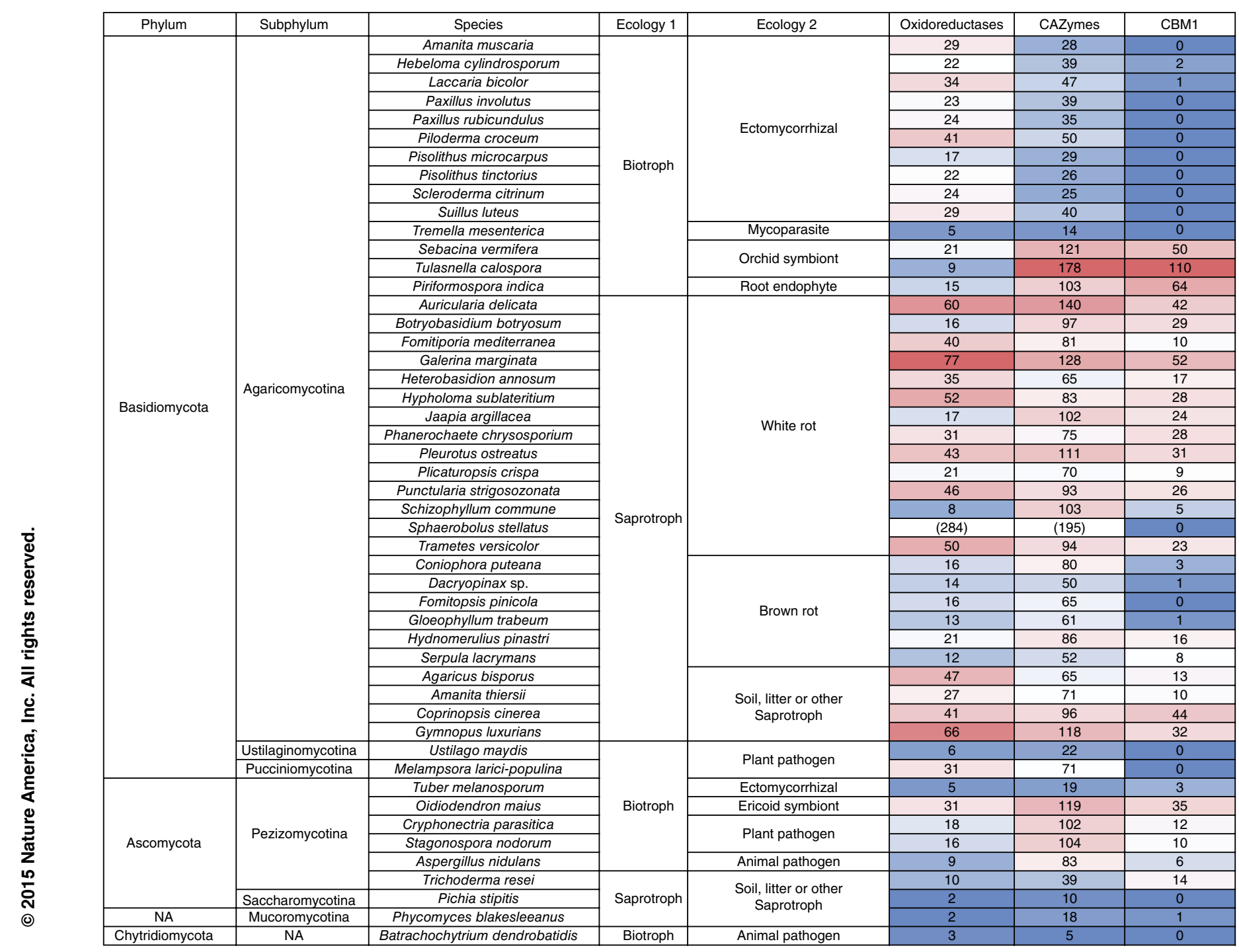

Figure 2 Summary of lignocellulose-degrading oxidoreductases, carbohydrate-active enzymes (CAZymes) and cellulose-binding modules in 49 fungal genomes. Relative abundance of genes is represented by a color scale, from the minimum (blue) to maximum (red) number of copies per species (excluding Sphaerobolus stellatus; values in parentheses). Individual gene families are shown in Supplementary Table 7. NA, not applicable.

gene, which are localized to the lineage leading to the ancestor of Auriculariales (Fig. 1). From that point, multiple copies of genes encoding LPMOs, PODs and GLXs, as well as GH6 and GH7, are reconstructed in the 'backbone' nodes of the Agaricomycete phylogeny (Fig. 1), thus suggesting that white rot was a conserved trait in the early evolution of mushroom-forming fungi.

Although all ECM species have reduced complements of PCWDEs (Figs. 1 and 2, and Supplementary Figs. 3 and 12-23), they have arisen from functionally diverse saprotrophic precursors and have retained distinct suites of PCWDEs. For example, within the Agaricales, the ECM Amanita muscaria is nested within a group of soil and litter saprotrophs, including Amanita thiersii, which lacks PODs, whereas another ECM species, Hebeloma cylindrosporum, is nested within a group of white-rot decayers that have multiple POD, GLX, GH6, GH7 and LPMO enzymes (Fig. 1 and Supplementary Figs. 12-16). A. muscaria has lost genes encoding GLXs, GH6 and GH7, and it has a reduced complement of LPMO genes; both may be consequences of a shift to ECM, but the loss of POD genes in A. muscaria preceded (and therefore could not have been caused by) the evolution of the ECM habit. In contrast, H. cylindrosporum has three POD genes encoding manganese peroxidases (Supplementary Fig. 4), thus suggesting that it may possess ligninolytic capabilities similar to those in Cortinarius glaucopus ${ }^{10}$.

The most densely sampled clade of ECM species in our analysis, the Boletales, contains six ECM species that are nested within a paraphyletic assemblage of brown-rot wood decayers lacking PODs and GLXs (Fig. 1). The ECM Boletales have further lost all copies of GH6 and GH7 genes and have no more than five copies of LPMO genes, thus suggesting that they have limited capacity to degrade lignocellulose (Fig. 1 and Supplementary Figs. 22 and 23). Gene tree-species tree reconciliations of LPMOs and other decay-related CAZymes suggest that parallel losses of brown-rot saprotrophy have occurred in the three ECM lineages in Boletales (Boletineae, Suillineae and Sclerodermatineae; Supplementary Figs. 15-23), although the ability to oxidize organic matter remains in Paxillus involutus 9 .

Cantharellales and Sebacinales are early-diverging lineages of Agaricomycetes that include the ORM symbionts Sebacina vermifera and Tulasnella calospora. In contrast to ECM taxa in Boletales and 


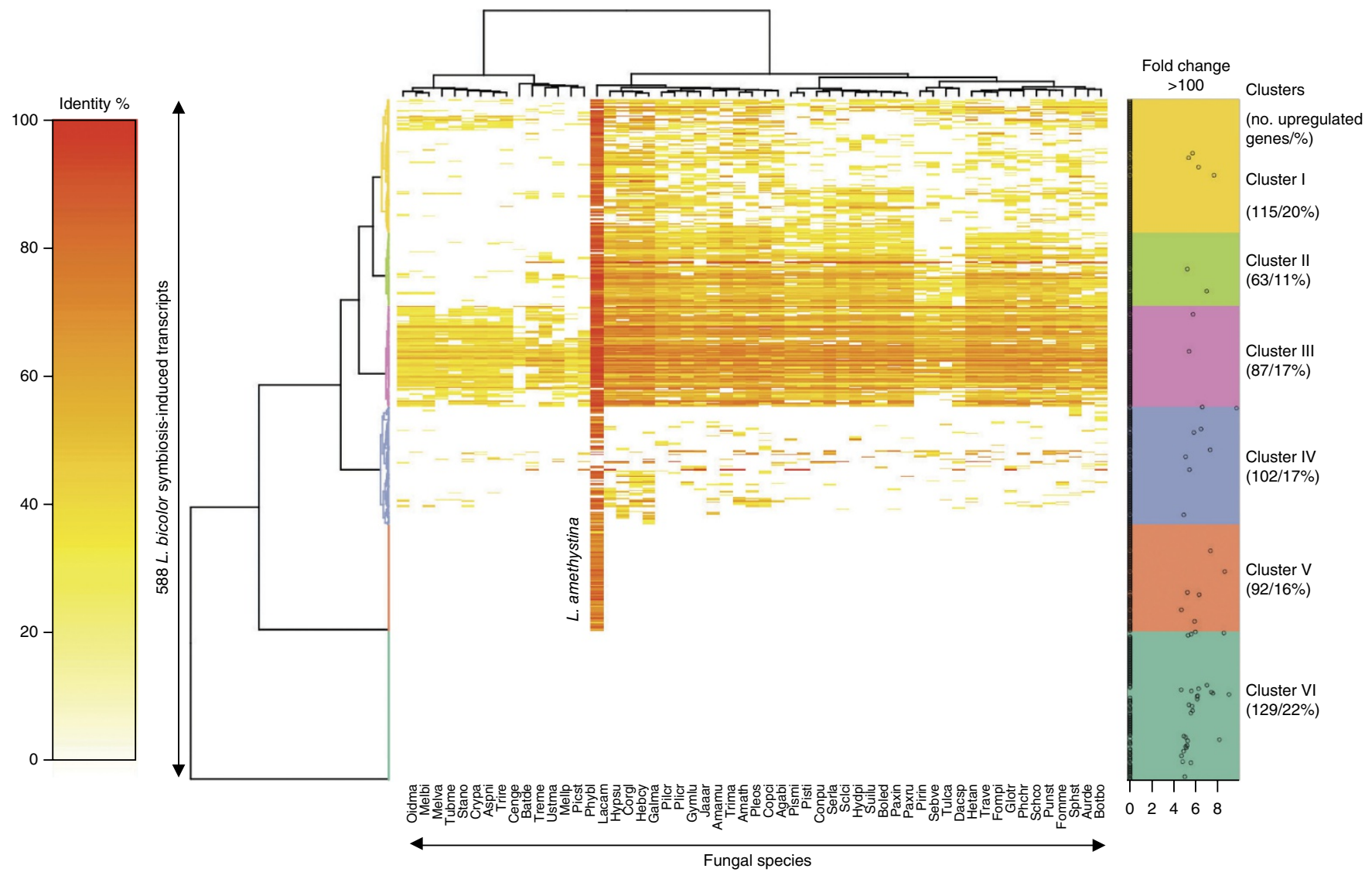

Figure 3 Presence and sequence similarity of symbiosis-upregulated genes from L. bicolor in 55 genomes of saprotrophic (white rots, brown rots, soil and litter decayers), mycorrhizal (ECM, ERM and ORM), pathogenic and endophytic fungi. The heat map depicts a double-hierarchical clustering of 588 symbiosis-upregulated $L$. bicolor genes (rows, fold change $>5$, false discovery rate-corrected $P<0.05$; Supplementary Data Set 1 ) based on their percentage sequence identity (color scale at left) with their orthologs (if any) in selected fungal species (columns). Right, color-coded gene clusters (clusters I to VI), with dots representing differentially expressed genes upregulated $>100$-fold; for genes with regulation less than 100-fold, the fold-change value is set to 1 (majority of the genes). The $x$ axis is a logarithmic scale of the fold change in gene expression (natural algorithm with Euler's number). Genes of cluster VI are L. bicolor-specific (orphan) genes, whereas genes of cluster $\mathrm{V}$ have sequence similarity only with predicted proteins of the sister species L. amethystina. Double-hierarchical-clustering heat maps of symbiosis-upregulated genes for the ECM A. muscaria,

H. cylindrosporum, P. involutus, P. croceum, S. luteus, the orchid mycorrhizal T. calospora, the ericoid mycorrhizal Oidiodendron maius, the endophytic S. vermifera and the brown-rot $S$. lacrymans are shown in Supplementary Figure 25, whereas double-hierarchical-clustering heat maps of symbiosisdownregulated genes are shown in Supplementary Figure 26. Species abbreviations are in Supplementary Table 1.

Agaricales, symbiotic members of Cantharellales and Sebacinales have a robust apparatus for degradation of crystalline cellulose, particularly T. calospora, which has 7 GH6, 27 GH7 and 33 LPMO genes (even more than its putatively saprotrophic sister taxon, B. botryosum) (Fig. 1 and Supplementary Figs. 15 and 16). Moreover, proteins with a cellulose-binding domain (CBM1) are rarely detected in ECM, whereas they are abundant in the ORM and ERM symbionts (Fig. 2 and Supplementary Table 7). Unlike typical ECM fungi, ORM fungi transfer carbohydrates to their hosts in the juvenile state of orchids, and they exploit nonliving organic substrates to feed their host ${ }^{16}$. Similarly, the ERM Oidiodendron maius (Ascomycota) maintains multiple copies of GH6, GH7 and LPMO genes (Fig. 1 and Supplementary Table 7), thus explaining its saprotrophic ability in Sphagnum peat ${ }^{17}$. Reconciliation analyses suggest that the divergence of Cantharellales and Sebacinales occurred before the diversification of PODs in Agaricomycetes and the origin of white rot (Fig. 1). The well-developed capacity to attack crystalline cellulose in these lineages and in the Ascomycota may reflect a primitive mode of symbiotic life substantially relying on the saprotrophic ability to decay nonwoody substrates with modest lignin content.
In $L$. bicolor, symbiosis requires lineage-specific genes encoding small secreted effector proteins that control host-plant development and immunity ${ }^{4,11,12}$. By sequencing RNA from free-living mycelia and mycorrhizal roots, we identified genes regulated by symbiosis development in the ECM A. muscaria, $H$. cylindrosporum, P. involutus, Piloderma croceum and Suillus luteus, the ERM O. maius and the ORM S. vermifera and T. calospora. Of the expressed genes, $1.3-6.0 \%$ are upregulated during symbiosis and $0.2-4.9 \%$ are downregulated by the interaction (fold change $>5$, false discovery rate-corrected $P<0.05$; Supplementary Note and Supplementary Fig. 24). Though genes belonging to similar functional gene ontology (GO) categories (signaling, information storage and processing, and metabolism) were upregulated in these interactions (Supplementary Fig. 25; downregulated genes in Supplementary Fig. 26), each species expressed a distinct set of genes thought to be involved in redox reactions, nutrient transport and metabolism. A large set of symbiosis-upregulated genes have orthologs in brown- and white-rot fungi (cluster III, Fig. 3), thus suggesting that they are not unique to mycorrhizal symbionts and tend to be associated with essential core metabolic pathways. These conserved mycorrhiza-induced genes might provide clues to how ancestral 


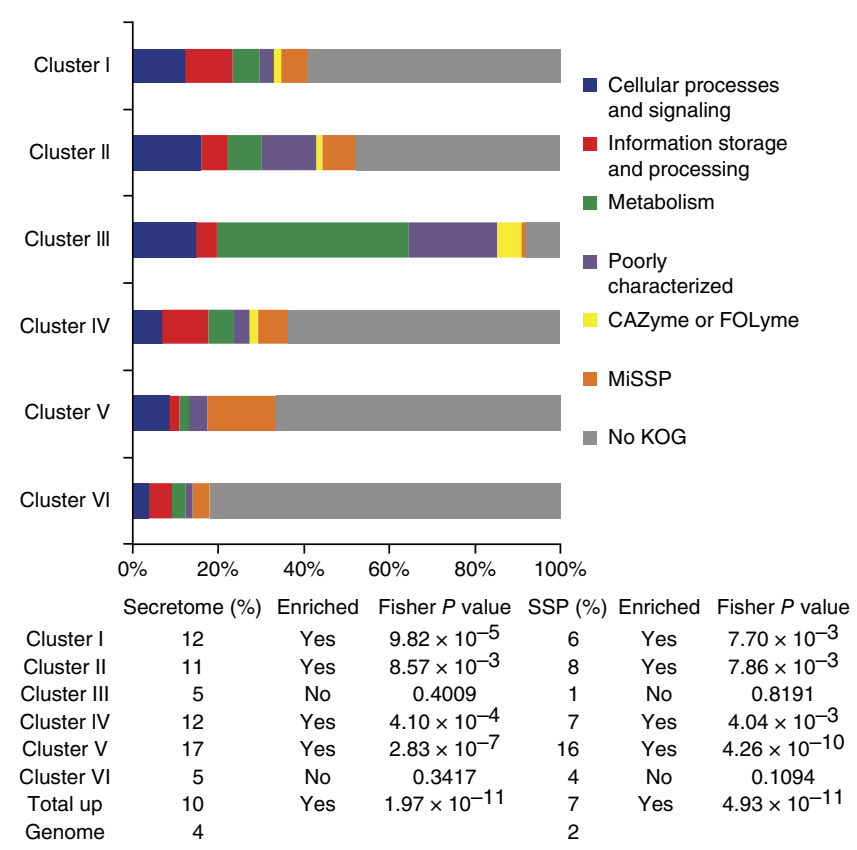

Figure 4 Distribution of symbiosis-upregulated genes of clusters I to VI into functional categories, including MiSSPs and nonsecreted orphan genes (no KOG). MiSSPs represent $16 \%$ of the symbiosis-upregulated transcripts in cluster $\mathrm{V}$, a significant enrichment compared to their percentage in the total gene repertoire (i.e., $2 \%$ ). CAZyme, carbohydrateactive enzymes; FOLyme, fungal oxidative lignin-degrading enzymes.

gene complements have been adapted to the ECM lifestyle. For example, the few retained PCWDEs acting on pectin (GH28, GH88 and CE8), hemicellulose (GH30) and cellulose (GH5_5 and LPMOs) are expressed in ECM root tips likely to modify the plant cell wall during colonization of the host root apoplastic space (Supplementary Fig. 27). In contrast, ORM and ERM symbionts expressed a full complement of PCWDEs in symbiosis (Supplementary Figs. 27 and 28), thus suggesting that they are used to penetrate host cells.

Many (7-38\%) of the symbiosis-induced genes are restricted to a single ECM species (clusters V and VI, Fig. 3; Supplementary Fig. 25), even in the densely sampled Boletales (23\% in S. luteus; Supplementary Fig. 25). Only one-third of the Laccaria symbiosisinduced orphan genes have homologs in both L. bicolor and Laccaria amethystina (Fig. 3). The latter diverged from the L. bicolor lineage $\sim 20$ million years ago ${ }^{18}$, thus indicating that even after the evolution of the ECM habit species within a genus continued to develop a specific symbiosis protein 'toolkit' and to diverge from each other. Lineagespecific orphan genes may represent either ancestral genes that have diverged so far that their similarities to other sequences have been obscured, a phenomenon frequently observed for effector genes ${ }^{19}$, or genes formed de novo from previously noncoding sequences ${ }^{20}$. Orphan fungal genes are over-represented among up- and downregulated genes found in mycorrhizal roots (Figs. 3 and 4, and Supplementary Figs. 25 and 26). To assess whether previously uncharacterized but highly induced genes may represent candidates for secreted effector proteins (CSEPs), we assessed which and how many of these genes encode proteins that possess properties (for example, predicted signal peptide, size $<300$ amino acid residues and no sequence similarity) common to known secreted effectors, such as mycorrhiza-induced small secreted protein of $7 \mathrm{kDa}$ (MiSSP7) ${ }^{11,12}$. Of the induced genes, $8-28 \%$ encode proteins with a predicted signal peptide, a significantly higher fraction than the $3-7 \%$ of noninduced and non-symbiosis-related genes that encode a protein with a signal peptide $\left(P=2.04 \times 10^{-6}\right.$ to $<2.20 \times 10^{-16}$, Fisher's exact test; Fig. 4 and Supplementary Fig. 25). Except for S. vermifera, there is a significantly higher proportion of genes encoding secreted proteins of $<300$ amino acid residues (4-11\% compared to $1-2 \%$ in the noninduced set; $P=0.00333$ to $<2.20 \times$ $10^{-16}$, Fisher's exact test; Fig. 4 and Supplementary Fig. 25).

Serpula lacrymans is a member of a paraphyletic clade of brownrot lineages from which the ECM Boletales are derived (Fig. 1). S. lacrymans is able to form a loose hyphal mantle around pine roots, and this may provide clues to the antecedent condition of ECM ${ }^{21}$. Transcript profiling of the root-associated mycelium showed a significant proportion of root-induced transcripts, but we detected no small secreted proteins, whereas ECM Boletales expressed a large set of MiSSPs (Supplementary Fig. 25). Although proteins known to interact with host immunity and development, such as MiSSP7, are not the only functional class to be over-represented among lineage-specific short secreted proteins, the prevalence of CSEPs among symbiosis-induced genes suggests that at least some of these genes encode new symbiosis-related effectors.

Mycorrhizal symbioses evolved from ecologically diverse decayer precursors and radiated in parallel, following the origins of their hostplant lineages (Fig. 1). Polyphyletic evolution of the ECM lifestyle is marked not only by convergent losses of different components of the ancestral saprotrophic apparatus but also by rapid genetic turnover in symbiosis-induced genes (Fig. 3), some of which may reflect lineage-specific functional innovations, such as MiSSPs. In contrast, ERM and ORM fungi retained an extensive decay apparatus that is probably exploited indirectly by the plant for carbohydrate supply, thus explaining their known saprotrophic ability ${ }^{17}$. The available genome sequences of mycorrhizal fungi will represent foundational information for understanding symbiosis development and functioning. These resources will facilitate field studies aiming to predict responses of mycorrhizal communities to environmental shifts, such as altered forest-management practices and climate change.

\section{METHODS}

Methods and any associated references are available in the online version of the paper.

Accession codes. Genome assemblies and annotations for the organisms used in this study are available via the JGI fungal genome portal MycoCosm 22 (Supplementary Tables 2 and 3). In addition, the newly sequenced genome assemblies and annotations have been deposited in GenBank under the following accession codes and BioProjects, respectively: A. muscaria Koide BX008, JMDV00000000 and PRJNA207684; Gymnopus luxurians FD-317 M1, JJNP00000000 and PRJNA68535; H. cylindrosporum h7, JMDQ00000000 and PRJNA207849; Hydnomerulius pinastri MD 312, JMSK00000000 and PRJNA207871; Hypholoma sublateritium FD-334 SS-4, JMSJ00000000 and PRJNA70685; L. amethystina LaAM-08-1, JMSL00000000 and PRJNA196025; O. maius Zn, JMDP00000000 and PRJNA74727; P. involutus ATCC 200175, JOMD00000000 and PRJNA60449; Paxillus rubicundulus Ve08.2h10, JMDR00000000 and PRJNA243391; P. croceum F 1598, JMDN00000000 and PRJNA61203; Pisolithus microcarpus 441, JMDM00000000 and PRJNA60815; Pisolithus tinctorius Marx 270, JMDO00000000 and PRJNA207840; Plicaturopsis crispa FD-325 SS-3, JOMB00000000 and PRJNA207847; Scleroderma citrinum Foug A, JMDU00000000 and PRJNA207859; S. vermifera MAFF 305830, JMDS00000000 and PRJNA207844; Sphaerobolus stellatus SS14, JOMA00000000 and PRJNA207858; S. luteus UH-SluLm8-n1, JMSM00000000 and PRJNA242126; T. calospora MUT 4182, 
JMDT00000000 and PRJNA207843). Electronic files of the phylogenomic and phylogenetic analyses have been deposited in the Dryad Repository under doi:10.5061/dryad.f2g0f. The complete gene expression data sets have been submitted to Gene Expression Omnibus (superseries GSE63947). For L. bicolor and S. lacrymans, microarray data $^{4,21}$ were reanalyzed (GSE9784, GSE27839 and GSE63929).

Note: Any Supplementary Information and Source Data files are available in the online version of the paper.

\section{ACKNOWLEDGMENTS}

This material is based on work conducted by the US Department of Energy Joint Genome Institute, a DOE Office of Science User Facility, supported under contract no. DE-AC02-05CH11231. This work was also supported by the Laboratory of Excellence ARBRE (ANR-11-LABX-0002-01), the Genomic Science Program (Plant-Microbe Interactions project) funded by the US Department of Energy, Office of Science, Biological and Environmental Research (contract DE-AC05-00OR22725), the Lorraine Region Council (to F.M.), US National Science Foundation grants DEB-1208719 and DEB-0933081 (both to D.S.H. and DEB-1021606 (to A.P.)), the German Centre for Integrative Biodiversity Research (iDiv) Halle-Jena-Leipzig (DFG FTZ 118) and the German Science Foundation (DFG, grant BU941/20-1) (to F.B.) and the Swedish Research Council (to A. Tunlid). This work was partly supported by the Laboratory of Excellence TULIP (ANR-10-LABX-41 and ANR-11-IDEX-0002-02). F.M. would like to acknowledge M.A. Selosse and B. Lindahl for helpful discussions.

\section{AUTHOR CONTRIBUTIONS}

F.M. conceived and guided the entire Mycorrhizal Genomics Initiative. I.V.G. directed all genome-sequencing and analysis efforts at JGI. D.S.H. directed the phylogenomic analyses. A. Tunlid coordinated the P. involutus genome project. F.M., D.S.H., I.V.G. and A. Kohler wrote the paper with contributions and input from all project members. A. Kohler performed overall management of biological materials and DNA and RNA preparations at INRA. K.W.B. performed overall project management at JGI. N.C., M.D.C., J.D., D.F., T.J., U.L., E. Martino, M.P., J.R. and M.T. extracted genomic DNA for sequencing. J.D., N.H., M.G., M.T., H.-R.K., S.H., U.L., E. Martino, U.N., J.M.P., T.J. and F.R. provided RNA for transcript profiling. A. Kohler, E. Morin, F.M., C.M., C.V.-F., G.G., S.H., S.P., M.G., A.Z., F.B., J.C., R.M. and A.P. analyzed genomic and transcriptomic data. C.C. and C.Y.N. performed genome sequencing. A. Clum, A. Copeland, K.L., A. Tritt and H.S. assembled the genomes. A. Kuo, R.A.O., R.R. and A.S. annotated and analyzed the genomes. B.C. and F.S. analyzed the P. involutus genome. E.A.L. and A. Lipzen performed transcriptome sequencing and analysis at JGI. L.G.N., D.F. and D.S.H. performed organismal phylogeny and analyzed evolution of decay-related gene families. A. Kuo and E. Morin performed protein clustering analysis. B.H. and A. Levasseur annotated the CAZyme and fungal oxidative lignin enzyme (FOLyme) families. J.H. analyzed the repeated elements in A. muscaria.

\section{COMPETING FINANCIAL INTERESTS}

The authors declare no competing financial interests.

Reprints and permissions information is available online at http://www.nature.com/ reprints/index.html.
This work is licensed under a Creative Commons AttributionNonCommercial-ShareAlike 3.0 Unported License. The images or other third party material in this article are included in the article's Creative Commons license, unless indicated otherwise in the credit line; if the material is not included under the Creative Commons license, users will need to obtain permission from the license holder to reproduce the material. To view a copy of this license, visit http://creativecommons. org/licenses/by-nc-sa/3.0/.

1. Högberg, M.N. \& Högberg, P. Extramatrical ectomycorrhizal mycelium contributes one-third of microbial biomass and produces, together with associated roots, half the dissolved organic carbon in a forest soil. New Phytol. 154, 791-795 (2002).

2. Read, D.J., Leake, J.R. \& Perez-Moreno, J. Mycorrhizal fungi as drivers of ecosystem processes in heathland and boreal forest biomes. Can. J. Bot. 82, 1243-1263 (2004).

3. Averill, C., Turner, B.L. \& Finzi, A.C. Mycorrhiza-mediated competition between plants and decomposers drives soil carbon storage. Nature 505, 543-545 (2014).

4. Martin, F. et al. Symbiosis insights from the genome of the mycorrhizal basidiomycete Laccaria bicolor. Nature 452, 88-92 (2008).

5. Martin, F. et al. Périgord black truffle genome uncovers evolutionary origins and mechanisms of symbiosis. Nature 464, 1033-1038 (2010).

6. Veneault-Fourrey, C. et al. Genomic and transcriptomic analysis of Laccaria bicolor CAZome reveals insights into polysaccharides remodelling during symbiosis establishment. Fungal Biol. Gen. 72, 168-181 (2014).

7. Clemmensen, K.E. et al. Roots and associated fungi drive long-term carbon sequestration in boreal forest. Science 339, 1615-1618 (2013).

8. Treseder, K.K., Torn, M.S. \& Masiello, C.A. An ecosystem-scale radiocarbon tracer to test use of litter carbon by ectomycorrhizal fungi. Soil Biol. Biochem. 38 1077-1082 (2006).

9. Rineau, F. et al. Carbon availability triggers the decomposition of plant litter and assimilation of nitrogen by an ectomycorrhizal fungus. ISME J. 7, 2010-2022 (2013).

10. Bödeker, I.T.M. et al. Ectomycorrhizal Cortinarius species participate in enzymatic oxidation of humus in northern forest ecosystems. New Phytol. 203, 245-256 (2014).

11. Plett, J.M. et al. A secreted effector protein of Laccaria bicolor is required for symbiosis development. Curr. Biol. 21, 1197-1203 (2011).

12. Plett, J.M. et al. The effector MiSSP7 of the mutualistic fungus Laccaria bicolor stabilizes the Populus JAZ6 protein and represses JA-responsive genes. Proc. Natl. Acad. Sci. USA 111, 8299-8304 (2014).

13. Smith, S.E. \& Read, J.R. Mycorrhizal Symbiosis (Academic Press, 2008).

14. Tedersoo, L., May, T.W. \& Smith, M.E. Ectomycorrhizal lifestyle in fungi: global diversity, distribution, and evolution of phylogenetic lineages. Mycorrhiza 20, 217-263 (2010).

15. Tisserant, E. et al. The genome of an arbuscular mycorrhizal fungus provides insights into the oldest plant symbiosis. Proc. Natl. Acad. Sci. USA 110, 20117-20122 (2013).

16. Dearnaley, J.D.W. Martos, F. \& Selosse, M.-A. in Fungal Associations 2nd edn. Vol. 9 (ed. Hock, B.) Ch. 12, 207-230 (Springer, 2012).

17. Rice, A.V. \& Currah, R.S. in Microbial Root Endophytes (eds. Schulz, B., Boyle, C. \& Sieber, T.) Ch. 13, 227-246 (Springer, 2006).

18. Ryberg, M. \& Matheny, B. Asynchronous origins of ectomycorrhizal clades of Agaricales. Proc. R. Soc. 279, 2003-2011 (2012).

19. Stergiopoulos, I. \& de Wit, P. Fungal effector proteins. Annu. Rev. Phytopathol. 47, 233-263 (2009).

20. Carvunis, A.R. et al. Proto-genes and de novo gene birth. Nature 487, 370-374 (2012).

21. Eastwood, D.C. et al. The plant cell-wall-decomposing machinery underlies the functional diversity of forest fungi. Science 333, 762-765 (2011).

22. Grigoriev, I.V. et al. MycoCosm portal: gearing up for 1000 fungal genomes. Nucleic Acids Res. 42, D699-D704 (2014). 


\section{ONLINE METHODS}

Sequencing, assembly and annotation. The 18 new genomes were sequenced with a combination of the Sanger, 454, Illumina and PacBio sequencing platforms (Supplementary Note and Supplementary Tables 2 and 3). All genomes were assembled in a platform-dependent manner and annotated with the JGI Annotation Pipeline ${ }^{23}$, which combines several gene prediction and annotation methods with transcriptomics data and integrates the annotated genomes into $\mathrm{MycoCosm}{ }^{22}$, a web-based fungal resource for comparative analysis (Supplementary Note).

Transcript profiling. Gene expression in mycorrhizal root tips and free-living mycelium from the ECM A. muscaria, H. cylindrosporum, P. involutus, P. croceum and S. luteus, the ERM O. maius and the ORM S. vermifera and T. calospora was assessed with RNA-seq (Supplementary Note and Supplementary Table 11).

Protein sequence clustering. Predicted protein sequences were clustered with the Markov Cluster Algorithm (MCL) program ${ }^{24}$, with an inflation parameter of 2.0 (Supplementary Note).
Phylogeny. From protein sequence clustering, we identified single-copy gene families ( 1,809 clusters) that contained genes for $>15$ species (Supplementary Note and Supplementary Fig. 1). We found 617 such clusters, 5 of which contained suspected nonorthologous genes and were excluded. The remaining 611 gene families were retained, and ambiguously aligned sites were removed by 3 different settings altogether (with GBlocks and PRANK posterior probabilities). The single-gene alignments were then concatenated to result in 3 data sets with 19,567 sites and 149 loci (PRANK, 1.0 exclusion threshold), 114,814 sites and 542 loci (PRANK, 0.95 exclusion threshold) and 34,323 sites in 259 loci (MAFFT + GBlocks) (Supplementary Fig. 2). We then inferred maximum-likelihood and Bayesian trees by using RAxML 7.2.8 and PhyloBayes 3.3, respectively. The inferred trees were largely congruent across data sets and analyses. The inferred maximum-likelihood tree (data set PR0.95; Supplementary Fig. 2) was used for subsequent analyses.

23. Grigoriev, I.V. et al. The genome portal of the Department of Energy Joint Genome Institute. Nucleic Acids Res. 40, D26 (2012).

24. Enright, A.J., Van Dongen, S. \& Ouzounis, C.A. An efficient algorithm for large-scale detection of protein families. Nucleic Acids Res. 30, 1575 (2002). 


\section{Corrigendum: Convergent losses of decay mechanisms and rapid turnover of symbiosis genes in mycorrhizal mutualists}

Annegret Kohler, Alan Kuo, Laszlo G Nagy, Emmanuelle Morin, Kerrie W Barry, Francois Buscot, Björn Canbäck, Cindy Choi, Nicolas Cichocki, Alicia Clum, Jan Colpaert, Alex Copeland, Mauricio D Costa, Jeanne Doré, Dimitrios Floudas, Gilles Gay, Mariangela Girlanda, Bernard Henrissat, Sylvie Herrmann, Jaqueline Hess, Nils Högberg, Tomas Johansson, Hassine-Radhouane Khouja, Kurt LaButti, Urs Lahrmann, Anthony Levasseur, Erika A Lindquist, Anna Lipzen, Roland Marmeisse, Elena Martino, Claude Murat, Chew Y Ngan, Uwe Nehls, Jonathan M Plett, Anne Pringle, Robin A Ohm, Silvia Perotto, Martina Peter, Robert Riley, Francois Rineau, Joske Ruytinx, Asaf Salamov, Firoz Shah, Hui Sun, Mika Tarkka, Andrew Tritt, Claire Veneault-Fourrey, Alga Zuccaro, Mycorrhizal Genomics Initiative Consortium, Anders Tunlid, Igor V Grigoriev, David S Hibbett \& Francis Martin Nat. Genet.; doi:10.1038/ng.3223; corrected online 9 March 2015

In the version of this article initially published online, the Online Methods indicated that 612 single-gene families were included in the phylogenetic analysis. However, one gene family was excluded because of significant topological conflict, and the total number of gene families included was therefore 611. The error has been corrected for the print, PDF and HTML versions of this article. 